\title{
Diagnostic of cardio-vascular disease with help of largest Lyapunov exponent of RR-sequences
}

\author{
Alexey N. Pavlov ${ }^{\text {a,* }}$, Natalia B. Janson ${ }^{\text {a }}$, Vadim S. Anishchenko ${ }^{\text {a }}$, \\ Vladimir I. Gridnev ${ }^{b}$, Pavel Ya. Dovgalevsky ${ }^{\text {b }}$ \\ ${ }^{a}$ Nonlinear Dynamics Laboratory, Department of Physics, Saratov State University, Astrakhanskaya St. 83, 410026 Saratov, Russia \\ ${ }^{\mathrm{b}}$ Scientific Research Institute of Cardiology at Saratov State Medical University, Chernyshevskogo St. 141, 410028 Saratov, Russia
}

Accepted 18 August 1998

Communicated by T. Kapitaniak

\begin{abstract}
We suggest to present a discrete sequence of cardiointervals in the form of a smooth time dependence and for the given time series compute the largest Lyapunov exponent. Processing the database with RR-intervals of patients suffering from coronary artery disease (CAD) has shown that the largest Lyapunov exponent can be a diagnostic criteria allowing one to distinguish between different groups of patients with more confidence than the standard methods for time series processing accepted in cardiology. (C) 2000 Elsevier Science Ltd. All rights reserved.
\end{abstract}

\section{Introduction}

In recent years there has been a growth of interest of specialists in nonlinear dynamics to the problems of diagnostics in medicine and biology [1-21]. Beginning with the works [1-6] more and more scientists turn to the analysis of chaotic dynamics of various phenomena including functioning of alive organisms. Such an approach opens wide possibilities to apply powerful tools of nonlinear dynamics for the purpose of diagnostics.

Signals of electric activity of a heart, namely, electrocardiograms (ECGs), attract the attention of many researchers. For the present moment a large amount of works is known devoted to the study of ECG which has been fulfilled by either specialists in medicine with the use of some knowledge of nonlinear dynamics or by the physicists not possessing fundamental knowledge in physiology but having a large experience in processing the signals of complex structure (e.g., [7-23]). A large number of publications in this area require some generalization (standardization) of the known methods for time series analysis to reveal the most informative ones which can be recommended for diagnostic [24].

At present the majority of the researchers prefer to deal not with the ECG but with the sequence of RRintervals, i.e., the time distances between the neighboring R-peaks. This fact can be explained as follows. First, keeping a database of ECGs requires much more computer memory than keeping of RR-intervals. Second, on the opinion of cardiologists, just RR-intervals contain information about a functional state of a cardio-vascular system of organism, while a typical PQRST-complex repeats itself at every heartbeat with

\footnotetext{
* Corresponding author.

E-mail address: pavlov@chaos.ssu.runnet.ru (A.N. Pavlov).
} 
minimal distortions. An important characteristics of ECG is heart rate variability, i.e., variation of RRintervals in time.

Well-known methods for the analysis of RR-intervals are given in the most complete form in the review [24]. The aim of the present research is to develop a technique for computing the largest Lyapunov exponent for a discrete sequence of cardiointervals and to experimentally prove the applicability of this method for the purposes of diagnostic of cardio-vascular system of a human.

\section{The ways of how to present RR-intervals}

For our task an important problem is how to present a sequence of RR-intervals. A traditional way is to plot the value of RR-interval versus the number of a heartbeat (Fig. 1). Such a dependence allows one to immediately evaluate the range of cardiointervals variation, build a probability density and compute some momental functions $\left(\overline{\mathrm{RR}}, \sigma_{\mathrm{RR}}^{2}\right)$. To perform spectral analysis a temporal axis is necessary, that is why usually the number $i$ is multiplied by the value of $\overline{\mathrm{RR}}$. As one works with short realizations ( $\leqslant 5 \mathrm{~min})$ for the better frequency resolution sometimes autoregressive methods or Fast Fourier Transform with different correlation windows are used.

Note that the traditional representation of RR-intervals (Fig. 1) seems to be rather artificial from the point of view of physics while another representation seems more reasonable (Fig. 2(b)). If all R-peaks are substituted by $\delta$-peaks (Fig. 2(b)) while all the other peaks are excluded from consideration one is able to compute the power spectrum analytically [25].

Since the value of RR-interval is determined with a certain accuracy which is defined by the sampling step $\Delta \mathrm{t}$ with which the ECG is recorded, the coordinate of $\delta$-peak is given with an error $\Delta \mathrm{t}$. That is why one can consider a sequence of rectangular impulses with the width $\Delta \mathrm{t}$ and finite amplitude (Fig. 2(c)) instead of $\delta$-peaks. For such a sequence one is able to analytically compute the power spectrum, too.

\section{Computing largest Lyapunov exponents of RR-sequences}

Spectral and correlation analysis are well-known and have been widely used for the processing of time series far before the concept of dynamical chaos has been established. Along with the latters, in recent years different entropies are being calculated for RR-intervals. Since computation of Shannon entropy $H=-\sum_{i=1}^{N} p_{i} \ln p_{i}$ for open systems is incorrect, the entropy should be normalized to the system's energy. Thus, a renormalized entropy $H_{p}=H / E$ can be used for RR-intervals, where $E$ is a magnitude proportional to total energy of the system in the frequency range meaningful for diagnostic or an entropy computed on the power spectrum and renormalized to the value $E-H_{\mathrm{s}}$ or other types of renormalized entropies $[19,20,26]$. The progress of nonlinear dynamics allows us to hope that new criteria can be developed which could be used in medical diagnostic.

At present the papers appear where besides the traditional methods of RR-intervals processing (computing momental functions, spectral analyzing, etc. [24]) correlation dimensions are computed [2,10] and

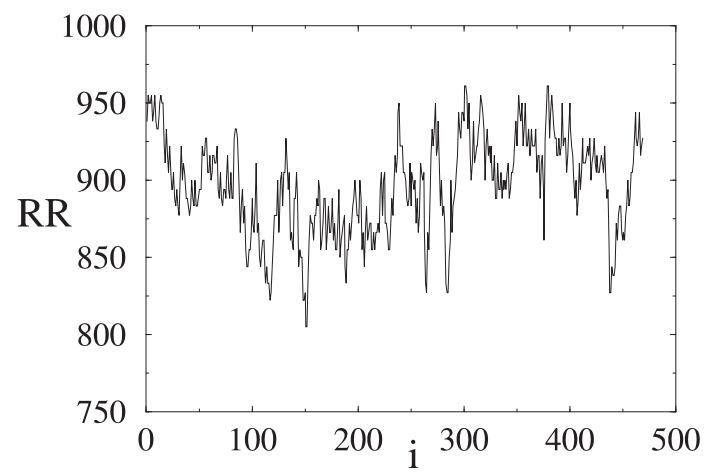

Fig. 1. Dependence of the value of RR-interval (in $\mathrm{ms}$ ) on the number of heart contraction. 


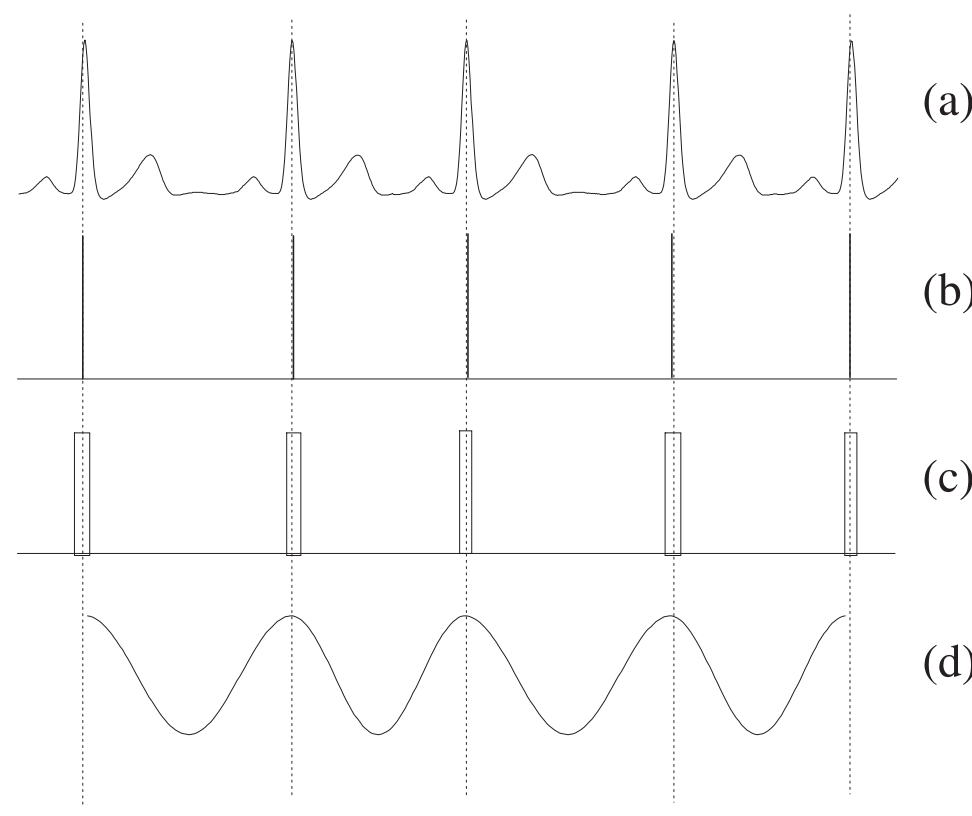

(a)

(b)

(c)

Fig. 2. Original ECG (2a) and different ways for presenting RR-intervals: as a sequence of $\delta$-functions (2b), sequence of rectangular impulses (2c) and a smooth time series (2d).

phase portraits are analyzed [11-13]. Kolmogorov entropy and Lyapunov exponents have not been evaluated from the short sequences of RR-intervals since one of the generally accepted methods [27] requires smooth signal.

To pass from the discrete signal to the continuous one may introduce the phase for the sequence of cardiointervals. At present different ways of how to introduce the phase are known [28]. The most simple way is to consider the time moments $t_{i}$ at which some events happen (e.g., crossing of the continuous signal of some threshold level). Supposing that the phase varies with respect to the linear law between these time moments introduce the following formula:

$$
\varphi=2 \pi \frac{t-t_{i}}{t_{i+1}-t_{i}} \pm 2 \pi i, \quad t_{i} \leqslant t<t_{i+1}
$$

Basing on this formula we suggest to transform the sequence of cardiointervals into a smooth temporal dependence (Fig. 2(d)):

$$
a(t)=\cos 2 \pi \frac{t-t_{i}}{\mathrm{RR}_{i}}, \quad t_{i} \leqslant t<t_{i+1},
$$

where $t_{i}$ and $t_{i+1}$ are the time moments corresponding to the appearance of R-peaks in the ECG.

Since the growth of phase in the interval between the two R-peaks is considered to be linear according to Eq. (1) and the segments of cosines with different periods are "sewed together" at the points $\varphi= \pm 2 \pi i$, the first derivative of the signal (2) does not suffer discontinuities. Thus, the suggested way of transition from a discrete series to a smooth signal (2) allows one to apply the method of largest Lyapunov exponent computation [27] to $a(t){ }^{1}$

\footnotetext{
${ }^{1}$ It is known [29,30], that one is not always able to compute the true value of largest Lyapunov exponent $\left(\lambda_{1}\right)$ on a one-dimensional realization, in general algorithm [27] allows one to define some characteristics $K$ of divergence of near-in-space phase trajectories or a measure of predictability which differs from the classical Lyapunov exponent. However, since for autonomous dynamical systems without noise forcing $K=\lambda_{1}$, this characteristics is associated with the largest LCE when the experimental data of biological origin are being processed [2], the latter being often not correct. In the present work we use the established terms and refer to the value computed by method [27] as to the Lyapunov exponent. The problem about the relation between $\lambda_{1}$ and $K$ requires a separate detailed investigation which we plan to realize in future.
} 
In the present paper we suggest one of the most simple ways of how to pass from a discrete signal to a smooth time dependence. Under transformation (2) the sequence of RR-intervals is transformed into a smooth dependence $a(t)$ being a signal with a complex frequency modulation. The main approximation of this model is the supposition that the instantaneous frequency is constant between the heart contractions. Our research corresponding to a more general case when we considered that the instantaneous frequency is the function of time [31] has shown that this supposition leads to an error in estimating the quantitative value of largest Lyapunov exponent. But the sign of it and its dependence on the regime of system's functioning is in good correspondence with the exact values computed for the case when the dependence of instantaneous frequency on time was taken into account. To prove everything stated above consider how the suggested "cosine method" works for a test models, namely, for modified oscillator with inertial nonlinearity (Anishchenko-Astakhov oscillator [32,33]):

$$
\frac{\mathrm{d} x}{\mathrm{~d} t}=m x+y-x z, \quad \frac{\mathrm{d} y}{\mathrm{~d} t}=-x, \quad \frac{\mathrm{d} z}{\mathrm{~d} t}=-g z+0.5 g(x+|x|) x
$$

and Rössler system [34]:

$$
\frac{\mathrm{d} x}{\mathrm{~d} t}=-(y+z), \quad \frac{\mathrm{d} y}{\mathrm{~d} t}=x+a y, \quad \frac{\mathrm{d} z}{\mathrm{~d} t}=b+z(x-c) .
$$

For such systems the analogue of RR-intervals can be introduced as the time distances between the successive maxima of some state variable (e.g., $x(t)$ ). We passed from the inter-maxima distances to the signal (2) and computed largest Lyapunov exponent $\lambda_{1}$ using the method [27] for different values of control parameters of the test models. Fig. 3 allows one to get sure that even if the largest LCE computed from the signal $a(t)$ (Fig. 3b,d) does not coincide with the true value of the exponent (Fig. 3a,c), it follows qualitatively the system's dynamics. Since when solving the diagnostics problem we are interested not in obtaining the exact value of Lyapunov exponent, but in establishing some objective criteria of different dynamical behavior, it is not of principal importance whether we compute the true value of LCE of some quantity which is proportional to it. Let us turn to experimental results.
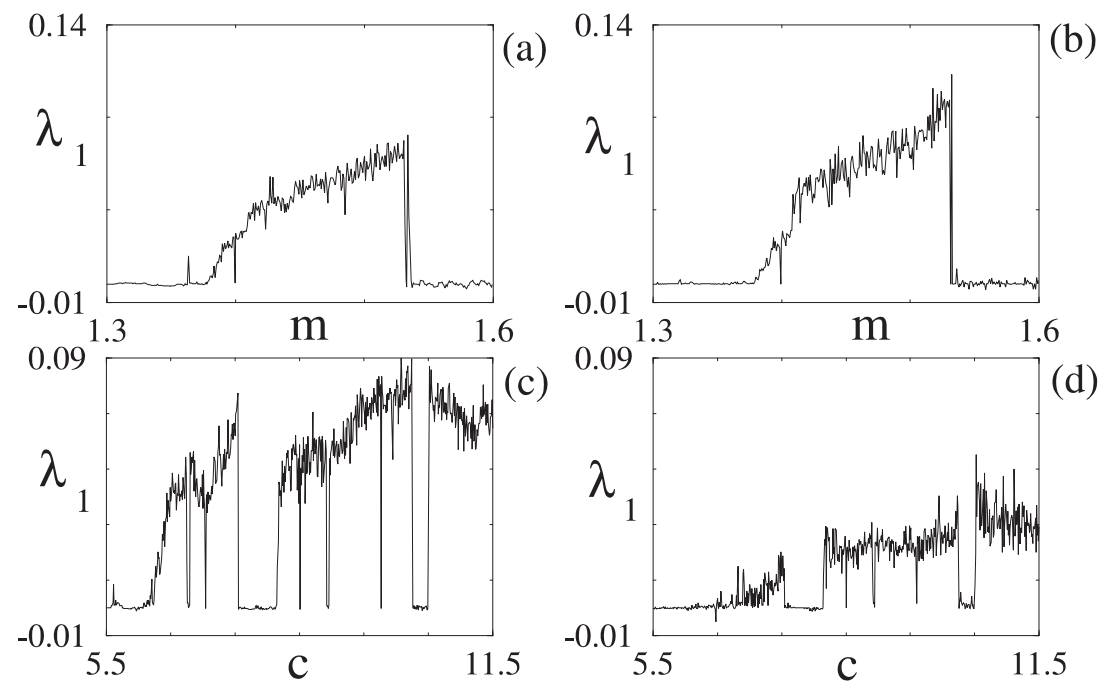

c)

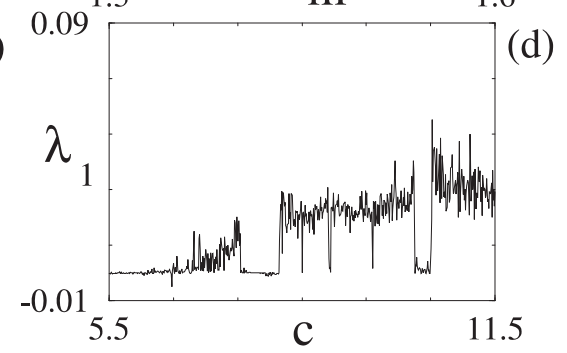

Fig. 3. Dependence of the value of Lyapunov characteristic exponent (LCE) on the values of control parameters of oscillator with inertial nonlinearity and Rössler system: $a, c$ are the true values of exponents for the systems (3) and (4), respectively; $b, d$ are the Lyapunov exponents computed from the signal shown in Fig. $2 \mathrm{~d}$ when the time intervals between the successive maxima of coordinate $x(t)$ were used as an analogue of RR-intervals. 


\section{Distinguishing between groups suffering from coronary artery disease of different degrees}

Forty patients of Saratov cardiocenter were included into the research suffering from coronary artery disease (CAD) of different degrees without clinical signs of heart disease. The basic methods for diagnostic of coronary artery disease (CAD) include:

- tolerance determination (the maximum admissible exercise which the patient is able to endure, i.e., the level of physical work-ability);

- Robinson index determination (characteristics showing the oxygen consumption by a myocard under

$75 \%$ of the physical exercise admissible for the age of the patient) and are based on such instrumental methods as coronarography, Holter monitoring of ECG, exercise test.

From the given list one can see that clinical methods for diagnostics of CAD are based on the series of criteria not including processing of RR-intervals. At the same time an important task of cardiology is the possibility of diagnostics just from the sequence of RR-intervals since they contain information about the state of the whole cardio-vascular system of an organism (this is a working hypothesis of cardiologists) and also their analysis does not require expensive equipment.

In the study all the patients (men) were divided into two groups according to the results of exercise test on CAD. The persons for whom the result of this test was positive formed the first group ( 24 people), while those for whom the result was negative formed the second group (16 people). The aim of our research is to reveal the criteria important for diagnostics which can be obtained from the processing of cardiointervals by means of very different methods for time series analysis.

The data bank available contain 80 files, two for each patient (with approximately $350-500$ point in each file.) The first recording of RR-intervals was made in the resting state, and the other one was made under loading of $25 \mathrm{Wt}$. The loading was as follows: the patient was turning the pedals of veloergometer with the velocity of approximately 60 turns per minute. After the velocity of turning had been stabilized (this was the end of transient process from the resting state to the loading) the sequence of cardiointervals was recorded during 5-7 min.

For all the time series from the data bank available a series of characteristics were computed which are described below. Using the terms of review [24], we considered the time domain and frequency domain characteristics: average value $(\overline{\mathrm{RR}})$; variance $\left(\sigma_{\mathrm{RR}}^{2}\right)$; energy in the range of lower frequencies (LF) [0.04, $0.15] \mathrm{Hz}\left(E_{\mathrm{LF}}\right)$; energy in the range of higher frequencies $(\mathrm{HF})[0.15,0.4] \mathrm{Hz}\left(E_{\mathrm{HF}}\right)$; the ratio of oscillation energies $\left(E_{\mathrm{LF}} / E_{\mathrm{HF}}\right)$. Along with the quantities mentioned above which are widely used in medicine we also used the following statistical and dynamical characteristics: entropy computed for the distribution of RRintervals and renormalized to the value which is proportional to the total energy of oscillations in the frequency range $\mathrm{LF}+\mathrm{HF}\left(H_{p}\right)$; entropy computed for the power spectrum and renormalized to the value which is proportional to the total energy of oscillations in the frequency range $\mathrm{LF}+\mathrm{HF}\left(H_{\mathrm{s}}\right)$; largest Lyapunov exponent computed by means of the algorithm [27] for the "cosine methods" suggested in this paper $\left(\lambda_{1}\right)$. The results of investigation of both groups are as follows (Table 1):

1. Only two characteristics, namely, normalized entropy computed from the power spectrum $\left(H_{\mathrm{s}}\right)$ and the largest Lyapunov exponent $\left(\lambda_{1}\right)$ allow one to distinguish between the two groups. ${ }^{2}$ In the first case the diagnostic criteria is the value of $H_{\mathrm{s}}$ under loading of $25 \mathrm{Wt}$ : in the first group 18 people from $24(75 \%)$ give $H_{\mathrm{s}} \leqslant 9.6$ while in the second group 11 persons from $16(\approx 69 \%)$ give $H_{\mathrm{s}} \geqslant 9.6$. Thus, the use of $H_{\mathrm{s}}$ allows us to identify correctly the membership of 29 persons from $40(\approx 72.5 \%)$ in one or another group.

In the second case, diagnostics is based on the reaction to the loading. In the first group for 17 of 24 persons $(\approx 70.8 \%)$ the largest LCE becomes less under loading, while in the second group it increases for the 12 persons from 16 ones $(75 \%)$. Computation of Lyapunov exponent allows us to identify also 29 persons from $40(\approx 72.5 \%)$.

All the other characteristics from those mentioned above do not give reliable diagnostics (namely, the probability of being associated with one group or another is less than $(65 \%)$.

\footnotetext{
${ }^{2}$ In cardiology distinguishing between the groups means that the selected criteria allows one to make diagnostics in more than $\frac{2}{3}$ of cases.
} 
Table 1

The results of cardiointervals processing for the patients suffering from coronary artery disease

\begin{tabular}{|c|c|c|c|c|c|}
\hline \multirow[t]{2}{*}{ State } & \multirow{2}{*}{$\begin{array}{l}\text { Group } \\
\text { number }\end{array}$} & \multirow{2}{*}{$\begin{array}{l}\text { The number of } \\
\text { patients in the group }\end{array}$} & \multicolumn{3}{|c|}{ The results of diagnostic using } \\
\hline & & & Variance, $\sigma_{\mathrm{RR}}^{2}$ & Entropy, $H_{\mathrm{s}}$ & $\begin{array}{l}\text { Lyapunov } \\
\text { exponent, } \lambda_{1}\end{array}$ \\
\hline \multirow[t]{2}{*}{ Resting } & 1 & 24 & 13 & 16 & $\begin{array}{l}\text { We investigate } \\
\text { response on the } \\
\text { loading }\end{array}$ \\
\hline & 2 & 16 & 8 & 8 & \\
\hline \multirow[t]{2}{*}{ Loading $25 \mathrm{Wt}$} & 1 & 24 & 15 & 18 & 17 \\
\hline & 2 & 16 & 9 & 11 & 12 \\
\hline
\end{tabular}

2. Computation of largest Lyapunov exponent is more preferable than computation of $H_{\mathrm{s}}$ since it gives a qualitative effect, namely, ordering or chaotization of oscillations under external influence (loading), while $H_{\mathrm{s}}$ is a quantitative criteria. A certain level of $H_{\mathrm{s}}$ is introduced conventionally. Also, since for 11 of 40 patients the values of $H_{\mathrm{s}}$ were obtained belonging to the interval $[9.5,9.7]$ a high precision of entropy computation is necessary, and, as a consequence, a high precision of power spectrum computation.

3. Finding Lyapunov exponent [27] assumes reconstruction of the phase portrait on the one-dimensional realization and the corresponding choice of the algorithm parameters (the embedding dimension, time delay $\tau$, etc.) [35-37]. Since for some patients the value of $\lambda_{1}$ varied slightly for the given parameter values we analyzed the dependence of $\lambda_{1}$ on parameters. In Table 1 the values of $\lambda_{1}$ are given for the fixed embedding dimension $N=5$. Each value of $\lambda_{1}$ is averaged over the results obtained for different values of $\tau$ so that for every value the reconstructed phase portrait does not stretch significantly in some selected direction. Such a procedure requires much more time than computation of all the other characteristics, but it allows to trust the obtained results to a larger extent.

4. Chaotization (or ordering) of oscillations under external forcing which can be detected by the increasing (or decreasing) of the value of $\lambda_{1}$ is accompanied by the signal's structure. Therefore, a question inevitably arises: why these changes were not detected by means of other computed characteristics, for example, power spectrum?
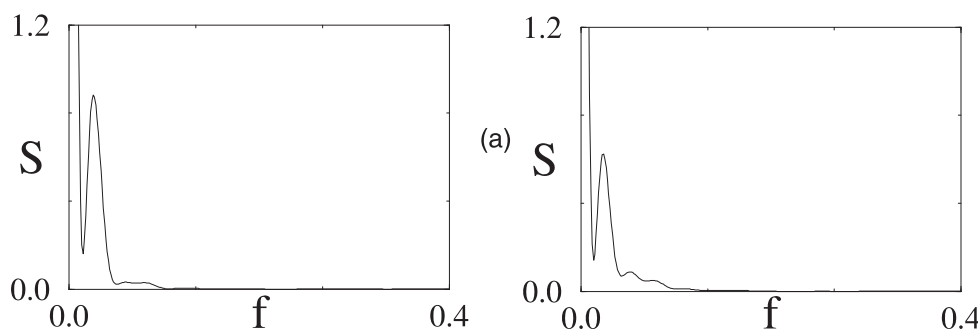

(b)
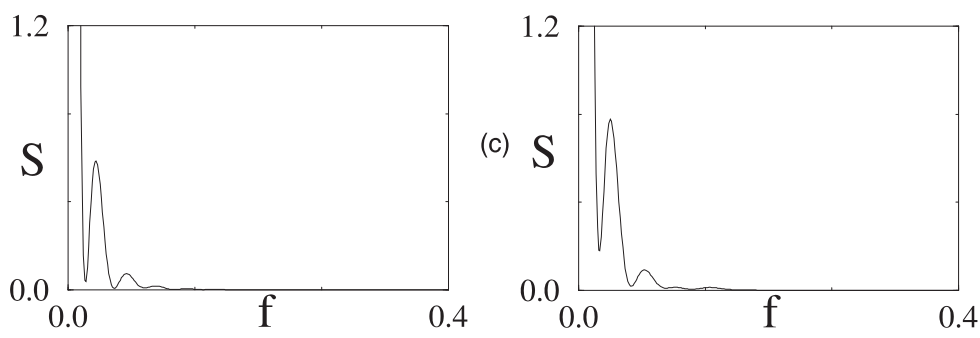

(d)

Fig. 4. The typical power spectra computed for the RR-sequences of patients suffering from CAD for both groups while resting (Fig. $4(\mathrm{a}, \mathrm{b}))$ and for loading of $25 \mathrm{Wt}($ Fig. $4(\mathrm{c}, \mathrm{d}))$. 
Our research has shown that the response to the loading is not identical for patients even from the same group. For a part of patients the increase of LCE is accompanied by the increase of the energy of oscillations in the higher frequency range (HF), while for the others the power spectrum is being varied in the range of ultra-low frequencies (ULF) $(f<0.04 \mathrm{~Hz})$. Taking into account the fact that computation in the range of ULF are not reliable for the short time series and also due to the absence of identical response to loading we were unable to establish a diagnostic tool using spectral analysis. Note that in agreement with the well-known standards of measurement [24], we took into account only a limited frequency range [0.0, $0.4]$ either for spectrum or for entropy computation. As is shown in Fig. 4, the power spectra computed for the RR-sequences of the patients of both groups are of the same type.

\section{Conclusions}

In the present work a method for RR-intervals presentation is suggested allowing one to apply to the given type of time series the algorithm for largest LCE computation. As our study has shown, for the given examples Lyapunov exponent may serve as a diagnostic tool allowing one to distinguish between the groups of patients more reliably than the standard for cardiology methods for time series analysis.

Our research includes the data measured from only 40 patients. Certainly, larger groups are necessary to verify the possibility of diagnostics according to the value of $\lambda_{1}$.

The advantage of the "cosine method" suggested in the present work is that it can be applied not only to RR-intervals, but also to a series of other data, for example, to "inter-spike intervals" (ISI) for neurons.

The transition from the sequence of discrete values to the smooth time dependence and the possibility to compute dynamical characteristics for signals generated by neurons allow us to hope that Lyapunov exponents will be added to the number of characteristics which are traditionally computed in recent years for the data of ISI type.

The current study was partly supported by INTAS grant 96-0305.

\section{References}

[1] A.L. Goldberger, B.J. West, Fractals in physiology and medicine, Yale J. Biol. Med. 60 (1987) 421.

[2] A. Babloyantz, A. Deslexhe, Is a normal heart a periodic oscillator, Biol. Cybernetics 58 (1988) 203.

[3] A.L. Goldberger, D.R. Rigney, B.J. West, Chaos and fractals in human physiology, Sci. Am. 262 (1990) 42.

[4] L. Glass, M.C. Mackey, From Clocks to Chaos: the rhythms of life, Princeton University Press, Princeton, NJ, 1988.

[5] L. Glass, Theory of Heart, Springer, New York, 1991.

[6] L. Glass, Cardiac arrhythmias and circle maps - a classical problem, Chaos 1 (1991) 13.

[7] C.K. Peng, J. Mietus, J.M. Hausdorff, S. Havlin, H.E. Stanley, A.L. Goldberger, Long-range anti-correlations and non-Gaussian behavior of the heartbeat, Phys. Rev. Lett. 70 (1993) 1343.

[8] C.K. Peng, S. Havlin, H.E. Stanley, A.L. Goldberger, Quantification of scaling exponents and crossover phenomena in nonstationary heartbeat time series, Chaos 5 (1) (1995) 82.

[9] C.K. Peng, S. Havlin, H.E. Stanley, A.L. Goldberger, Fractal scaling properties in nonstationary heartbeat time series, in: R.A. Katz (Ed.), Chaotic, Fractal and Nonlinear Signal Processing, Mystic, Juli, 1995, AIP Conf. Proc., vol. 375, AIP Press, New York, p. 615 .

[10] T.W. Frison, C.K. Peng, A. Goldberger, R.A. Katz, Chaotic data analysis of heart R-R interval EKG data, in: R.A. Katz (Ed.), Chaotic, Fractal and Nonlinear Signal Processing, Mystic, Juli, 1995, IP Conf. Proc., vol. 375, AIP Press, New York, p. 658.

[11] J. Ulbikas, A. Cenys, D. Zemaityte, G. Varoneskas, Correlations in heart beat data as quantitative characterization of heart pathology, in: R.A. Katz (Ed.), Chaotic, Fractal and Nonlinear Signal Processing, Mystic, Juli, 1995, AIP Conf. Proc., vol. 375, AIP Press, New York, p. 669.

[12] J.J. Zebrowski, W. Poplawska, R. Baranowski, Pattern entropy a tool for nonlinear dynamical analysis of a biological nonstationary system: the human heart, Acta Physica Polonika B 26 (6) (1995) 1055.

[13] R. Baranowski, J.J. Zebrowski, W. Poplawska, et al., Three-dimensional Poincare plots of the QT intervals - an approach to nonlinear QT analysis, Computers in Cardiology, 1995, p. 789.

[14] J.T. Bigger, J.L. Fleiss, R.C. Steinman, L.M. Rolnitzky, R.E. Kleiger, J.N. Rottman, Correlations among time and frequency domain measures of heart period variability two weeks after acute myocardial infarction, Am. J. Cardiology 69 (4) (1992) 891.

[15] M. Rosenblum, J. Kurths, A model of neural control of the heart rate, Physica A 215 (1995) 439.

[16] H. Seidel, H. Herzel, In: E. Mosekilde, O.G. Mouritsen (Eds.), Modelling the Dynamics of Biological Systems, Springer, Berlin, 1995, p. 205. 
[17] A. Bezerianos, T. Bountis, G. Papaioannou, P. Polydoropoulos, Nonlinear time series analysis of electrocardiograms, Chaos 5 (1) (1995) 95.

[18] A. Garfinkel, M.L. Spano, W.L. Ditto, J.N. Weiss, Controlling cardiac chaos, Science 257 (8) (1992) 1230.

[19] J. Kurths, A. Voss, P. Saparin, A. Witt, H.J. Kleiner, N. Wessel, Quantitative analysis of heart rate variability, Chaos 5 (1) (1995) 88.

[20] A. Voss, J. Kurths, H.J. Kleiner, A. Witt, P. Saparin, R. Dietz, H. Fiehring, N. Wessel, Neue methoden für die erkennung von hochrisikopatienten in der kardiologie, Biomedizinische Technik 39 (6) (1994) 134.

[21] P.I. Saparin, M.A. Zaks, J. Kurths, A. Voss, V.S. Anishchenko, Reconstruction and structure of electrocardiogram phase portraits, Phys. Rev. E 54 (1) (1996) 737.

[22] V.S. Anishchenko, N.B. Janson, A.N. Pavlov, Saddle focus in a model of the electrical activity of the human heart, Tech. Phys. Lett. 22 (2) (1996) 168.

[23] N.B. Janson, A.N. Pavlov, V.S. Anishchenko, One method for restoring inhomogeneous attractors, Int. J. Bif. Chaos 8 (4) (1998) 825.

[24] Task Force of the European Society of Cardiology and the North American Society of Pacing and Electrophysiology, Heart Rate Variability, Standards of Measurement, Physiological Interpretation and Clinical Use, Circulation 93 (5) (1996) 1043.

[25] R.W. DeBoer, J.M. Karemaker, J. Strackee, Comparing spectra of a series of point events particularly for heart rate variability data, IEEE Trans. Biomed. Eng. 31 (4) (1984) 384.

[26] Yu.L. Klimontovich, Turbulent Motion and the Structure of Chaos, Kluwer Academic Publishers, Dordrecht, 1991.

[27] A. Wolf, J.B. Swift, H.L. Swinney, J.A. Vastano, Determining Lyapunov exponents from a time series, Physica D 16 (1985) 285.

[28] A.S. Pikovsky, M.G. Rosenblum, G.V. Osipov, J. Kurths, Phase synchronization of chaotic oscillators by external driving, Physica D 104 (1997) 219.

[29] G. Paladin, M. Serva, A. Vulpiani, Complexity in dynamical systems with noise, Phys. Rev. Lett. 74 (1) (1995) 66.

[30] V. Loreto, G. Paladin, A. Vulpiani, Concept of complexity in random dynamical systems, Phys. Rev. E 53 (3) (1996) 2087.

[31] N.B. Janson, A.N. Pavlov, A.B. Neiman, V.S. Anishchenko, Reconstruction of dynamical and geometrical properties of chaotic attractors from threshold-crossing interspike intervals, Phys. Rev. E 58 (1) (1998) R4.

[32] V.S. Anishchenko, V.V. Astakhov, Bifurcations and chaos in an oscillator with inertial nonlinearity, in: T. Carroll, L. Pecora (Eds.), Nonlinear Dynamics in Circuits, World Scientific, Singapore, 1995, p. 55.

[33] V.S. Anishchenko, in: L. Chua (Ed.), Dynamical Chaos - Models and Experiments, World Scientific, Singapore, 1995.

[34] O.E. Rössler, An equation for continuous chaos, Phys. Lett. A 57 (1976) 397.

[35] N.H. Packard, J.P. Crutchfield, J.D. Farmer, R.S. Shaw, Geometry from a time series, Phys. Rev. Lett. 45 (1980) 712.

[36] F. Takens, Detecting strange attractors in turbulence, in: D.A. Rang, L.S. Young (Eds.), Lecture Notes in Mathematics, Warwick, 1980, vol. 898, Springer, Berlin, 1981, p. 366.

[37] J.L. Breeden, N.H. Packard, A learning algorithm for optimal representation of experimental data, Int. J. Bif. Chaos 4 (2) (1994) 311. 